\title{
Phosphorylation of $\alpha$-synuclein is crucial in compensating for proteasomal dysfunction
}

\author{
Hee soon Choi ${ }^{\mathrm{a}, 1}$, Hyunjeong Liew ${ }^{\mathrm{a}, 1}$, Ahram Jang ${ }^{\mathrm{a}}$, Yun-Mi Kim ${ }^{\mathrm{a}}$, Hilal Lashuel ${ }^{\mathrm{b}}$, Yoo-Hun Suh ${ }^{\mathrm{a}, *}$ \\ ${ }^{a}$ Department of Pharmacology, College of Medicine, Seoul National University, Seoul 110-799, South Korea \\ ${ }^{\mathrm{b}}$ Laboratory of Molecular Neurobiology and Neuroproteomics, Brain and Mind Institute, Ecole Polytechnique Federale de Lausanne, CH-1015 Lausanne, Switzerland
}

\section{A R T I C L E I N F O}

\section{Article history:}

Received 20 June 2012

Available online 7 July 2012

\section{Keywords:}

$\alpha$-Synuclein

Phosphorylation

HSC70

Lysosome

Proteasome

\section{Introduction}

$\alpha$-Synuclein ( $\alpha$-SYN) exists in presynaptic nerve terminals and is an intrinsically unfolded, acidic neuronal protein composed of 140 amino acids [1]. $\alpha$-SYN can normally be degraded by the ubiquitin-proteasomal system (UPS) and chaperone-lysosomal system (CLS). A number of studies have verified that both of these pathways are relevant in the clearance of $\alpha$-SYN [2-5].

Recently, the importance of the UPS in the neuron has been highlighted, and many researchers are interested in understanding the role of proteolytic systems in neurodegenerative disease. Since neuronal intracellular inclusions consist of ubiquitin-attractive protein aggregates that are thought to be a common characteristic of several neurodegenerative diseases, it has been considered that the failure of the UPS is the etiological cause of many neurodegenerative diseases [6]. Specifically, it was reported that aggregates of $\alpha$-SYN can be removed by the CLS [3] and to induce this phenomenon, $\alpha$-SYN must bind to heat shock proteins. We wondered

\footnotetext{
Abbreviations: PD, Parkinson's disease; HSC70, heat shock cognate 70; PTP1B, protein tyrosine phosphatase 1B; FAK, focal adhesion kinase; UPS, Ubiquitinproteasomal system; CLS, Chaperone-lysosomal system; CMA, chaperone-mediated autophagy; PQ, paraquat.

* Corresponding author. Fax: +82 27457996.

E-mail address: yhsuh@snu.ac.kr (Y.-H. Suh).

1 These authors contributed equally to this study.
}

which factor in $\alpha$-SYN is influenceable for binding with heat shock proteins.

$\alpha$-SYN can be post-translationally modified by phosphorylation [7,8], oxidation [9], nitration [10,11], sumoylation [12], and ubiquitination $[13,14]$. Several studies have shown that $\alpha$-SYN can be phosphorylated on serine or tyrosine residues $[15,16]$. Moreover, in other studies, functions of phosphorylation were suggested that phosphorylation of some serine residues is related to changes in $\alpha$ SYN localization [17], and tyrosine phosphorylation on residue 125 inhibits toxic oligomer formation and protects neurons from neurotoxicity in a PD Drosophila model, while serine phosphorylation has oligomer-promoting effects [18].

In our study, we demonstrated that the induction and continuous increase of $\alpha$-SYN phosphorylation on $\alpha$-SYN tyrosine residues, especially Y136, can accelerate its degradation. Furthermore, an increase in CLS activity in a model for neurodegenerative diseases effectively enhanced the degradation of misfolded or aggregated $\alpha-S Y N$ and reduced cellular toxicity.

\section{Materials and methods}

\subsection{Materials}

MG132, PTP1B inhibitor (3-(3,5-dibromo-4-hydroxy-benzoyl)2-ethyl-benzofuran-6-sulfonic acid-(4-(thiazol-2-ylsulfamyl- 
phinyl)- amide inhibitor) were purchased from Calbiochem (CA, USA), and 1,1'-Dimethyl-4-4'-bipyridinium dichloride hydrate (paraquat, PQ) was purchased from Sigma Aldrich (MO, USA). To silence the gene encoding FAK, siRNA was purchased from Santa Cruz Biotechnology (CA, USA). For immunoprecipitation and detection of tyrosine phosphorylated $\alpha$-synuclein, anti- $\alpha$-SYN (211), anti-pY20, anti-HSC70, anti-ubiquitin (Ub), anti- focal adhesion kinase pp125 (FAK), anti-p-FAK (925) antibodies and all of the HRP-conjugated antibodies were purchased from Santa Cruz Biotechnology (CA, USA). Alexa Fluor 488 goat-anti-mouse IgG and LysoTracker ${ }^{\circledR}$ were purchased from Invitrogen (CA, USA). The ubiquitination Kit was purchased from Biomol International (PA, USA).

\subsubsection{In vitro ubiquitination assay}

For the assay, ubiquitin, Mg-ATP, E1, E2 enzymes such as UB$\mathrm{CH} 5 \mathrm{a}, \mathrm{UBCH} 5 \mathrm{~b}$, and UBCH8, SH-SY5Y cell lysate for the E3 ligase and histidine-tagged recombinant $\alpha$-synuclein were mixed gently and incubated at $37^{\circ} \mathrm{C}$ for $1 \mathrm{~h}$. For Western blotting, the mixtures were quenched to terminate the reaction. For precipitation with $\mathrm{Ni}-\mathrm{NTA}$ super flow beads (Qiagen, CA, USA), the reaction was incubated with beads and $\mathrm{SH}-\mathrm{SY} 5 \mathrm{Y}$ cell lysate and rotated at $4{ }^{\circ} \mathrm{C}$ for $30 \mathrm{~min}$. The samples were analyzed by Western blotting.

\subsection{GST pull-down assays}

Each of the four tyrosine residues in $\alpha$-SYN was mutated to alanine using the KOD-Plus-Mutagenesis Kit (TOYOBO, Osaka, Japan). The point-mutated $\alpha$-SYN (Y39A, Y125A, Y133A, Y136A) was also expressed in TKB1-competent cells (Stratagene, CA, USA). GST pull-downs and Western blot analyses were performed with the wild-type and point-mutated GST- $\alpha$-SYN.

\subsection{Behavioral testing using the rotarod}

Eight-to 10-week-old male (20-25 g) C57BL6 mice were treated with paraquat $(\mathrm{PQ}, 30 \mathrm{mg} / \mathrm{kg}$ ) by a single intraperitoneal injection (i.p.) once a week for 3 weeks. The PTP1B inhibitor was dissolved in 95\% EtOH and diluted with PBS and administered by i.p. injection at a dose of $1 \mathrm{mg} / \mathrm{kg}$ every 2 days and $3 \mathrm{~h}$ before the PQ injection for 3 weeks.
A
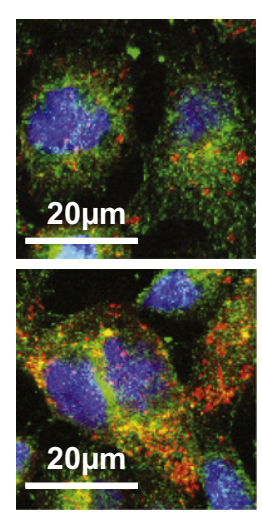

B

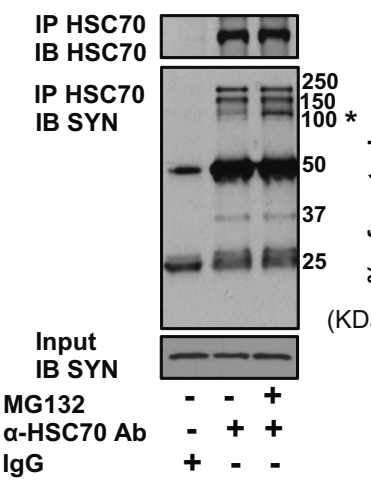

C



IB His IB Ub

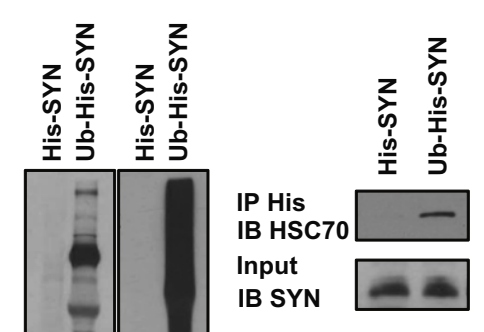

D

E

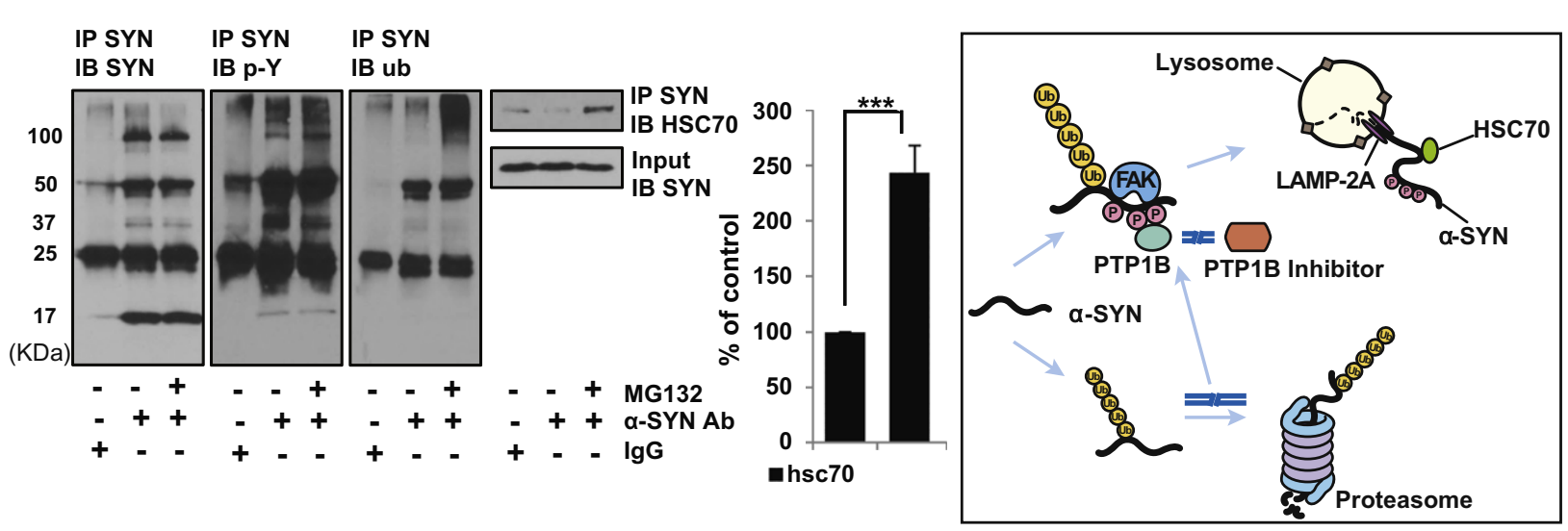

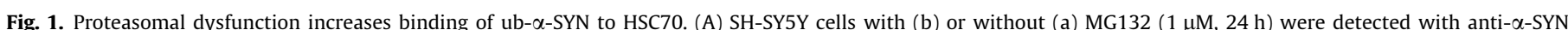

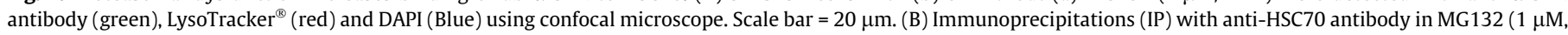

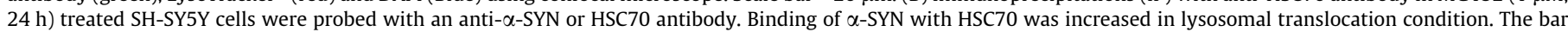

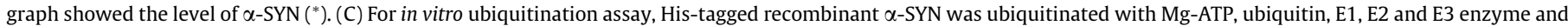

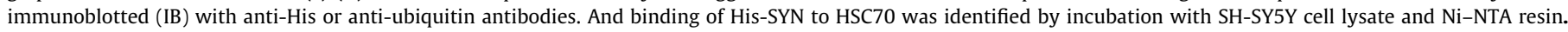

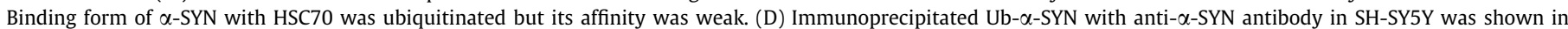

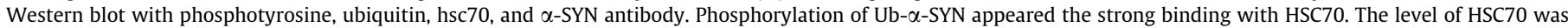
represented by bar graph. (E) Schematic diagram of turnover between proteasomal and lysosomal degradation of $\alpha$-SYN. $P<0.01(* *), P<0.001(* * *)$. 
Motor coordination and fatigue resistance of the PD mouse model were measured using an accelerating rotarod. Mice were conditioned three times at a speed of $8 \mathrm{rpm}$ for $1 \mathrm{~min}$. Thirty minutes after the last training, the locomotor activity of the mice was tested. The rotarod test was started by placing the mouse on a rod and was stopped when the mouse fell off the rod or after $300 \mathrm{~s}$ had passed, whichever came first. The rotation speed was gradually accelerated from 4 to $40 \mathrm{rpm}$. Each mouse was subjected to 5 trials per day with a 5 min interval between trials. The mice were divided into 3 groups with 7 animals in each group. Mice were housed one per cage on a 12:12 reversed light-dark cycle under controlled temperature $\left(25^{\circ} \mathrm{C}\right)$ with proper humidity and free access to food and water.

\subsection{Ethics statement}

The animals used in this experiment were handled according to the Guidelines for Animal Experimentation. This study was approved by the Ethics Committee of Seoul National University Institutional Animal Care and Use (SNU IACUC; approval ID; SNU-110105-4).

\subsection{Statistical analysis}

The Student's $t$-test and one-way ANOVA were performed using PASW statistics 18 (SPSS Inc., Chicago, IL, USA) to determine the relationships among the groups. Values of $P<0.05$ were considered to be significant.

\section{Results}

\subsection{Proteasomal dysfunction increases the binding of $\alpha$-SYN to HSC70}

To verify the changes in the location of $\alpha$-SYN, SH-SY5Y cells were treated with $1 \mu \mathrm{M}$ of MG132 for $24 \mathrm{~h}$. The cells were then stained with anti- $\alpha$-SYN (green) antibody. To determine the lysosomal stability and CLS activation, the cells were co-stained with LysoTracker $^{\circledR}$ (red). Under conditions of proteasomal dysfunction, lysosomes co-localized with $\alpha$-SYN were increased (Fig. 1A). This result suggests that degradation via the lysosomal pathway is activated and that $\alpha$-SYN is translocated into the activated lysosome, resulting from a blockage of the proteasomal degradation pathway.

We also found that the binding between HSC70 and $\alpha$-SYN was increased by treatment with $1 \mu \mathrm{M}$ MG132 using immunoprecipitation by anti-HSC70 antibody. That binding was increased in the proteasome dysfunctional condition and the binding form of $\alpha$-SYN was high molecular weight (Fig. 1B).

Therefore, to identify the status of high molecular weight $\alpha$-SYN, we performed the in vitro ubiquitination assay and we observed that ubiquitination of $\alpha$-SYN increased its binding to HSC70. His-tagged $\alpha$-SYN was ubiquitinated and precipitated by Ni-NTA resin after incubation with $\mathrm{SH}-\mathrm{SY} 5 \mathrm{Y}$ cell lysate. Consequentially, we identified that the amount of HSC70 bound to $\alpha$-SYN was

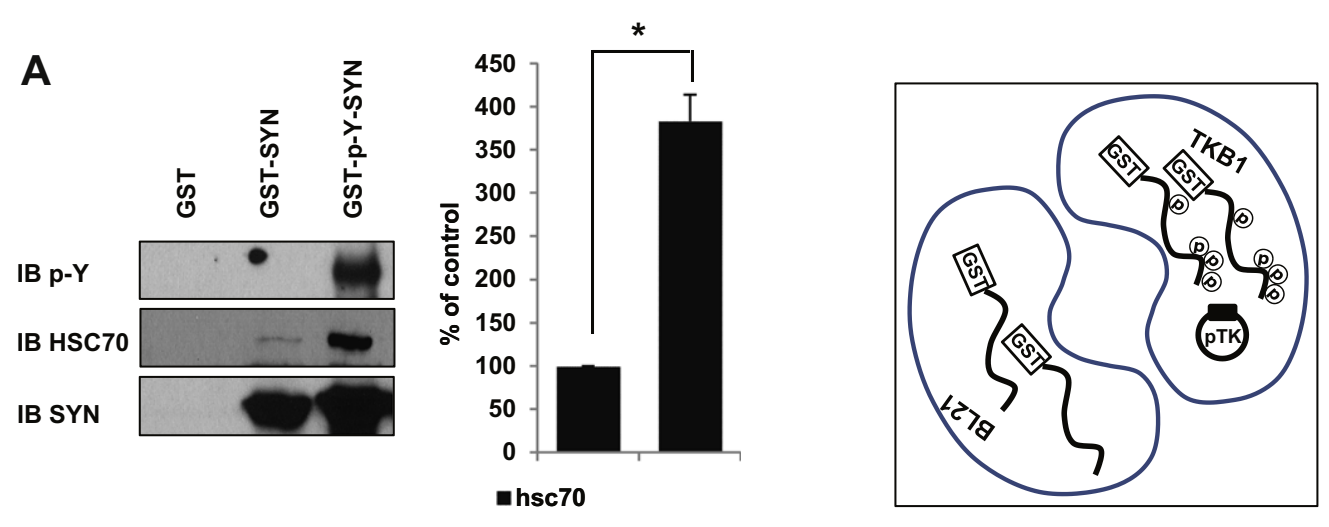

B

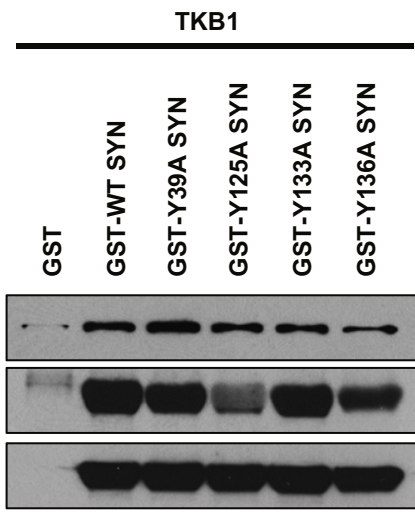

C

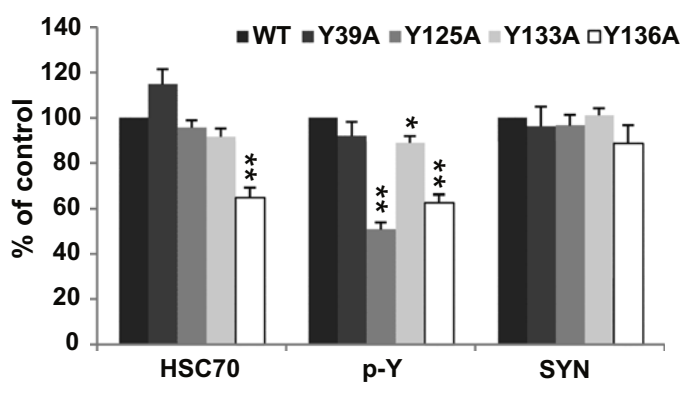

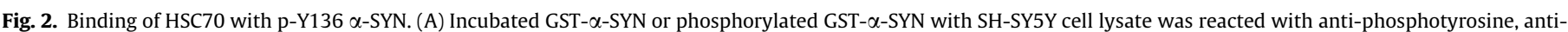

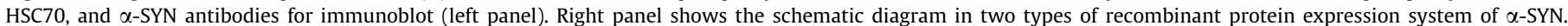



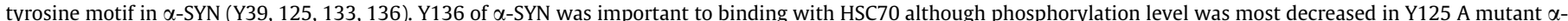

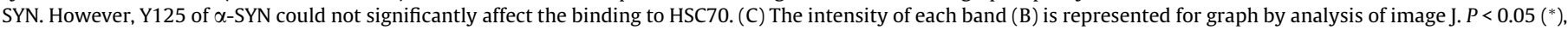
$\left.P<0.01{ }^{(* *}\right)$. 
increased when it was ubiquitinated (Fig. 1C). Moreover, we confirmed that the level of shifted $\alpha$-SYN, corresponding to ubiquitination, and the level of HSC70 bound to $\alpha$-SYN were increased with $1 \mu \mathrm{M}$ MG132 treatment using immunoprecipitaion by anti- $\alpha-S Y N$ antibody. And the phosphorylation of ubiquitinated $\alpha$-SYN was increased when the proteasome was not functional. (Fig. 1D).

\subsection{Tyrosine phosphorylation is most effective in promoting its} binding of HSC70 to $\alpha$-SYN

To investigate the factors that are important for binding $\alpha$-SYN and HSC70, tyrosine-phosphorylated GST- $\alpha$-SYN and nonphosphorylated GST- $\alpha$-SYN were prepared and used in a GST pull-down assay. GST- $\alpha$-SYN was expressed from the pGEX-4T1 plasmid vector in transformed competent cells. BL21- and TKB1-competent cells were used to express the nonphosphorylated and phosphorylated forms, respectively. Tyrosine phosphorylation of $\alpha$-SYN can be induced in TKB1 competent cells containing the plasmid of tyrosine kinase (Fig. 2A right). Nonphosphorylated or tyrosine phosphorylated $\alpha$-SYN were purified from bacterial cell lysates with GST beads and then incubated with SH-SY5Y cell lysate. The binding of HSC70 to tyrosine-phosphorylated $\alpha$-SYN was higher relative to its binding to nonphosphorylated $\alpha$-SYN. The level of HSC70 bound to $\alpha$-SYN was quantified using Image J (Fig. 2A left).

Next, we determined which of the four tyrosine residues (Tyr39, Tyr125, Tyr133 and Tyr136) in $\alpha$-SYN was most effective at promoting the binding to HSC70. Each tyrosine residue was mutated to alanine using the KOD-Plus-Mutagenesis Kit, and we performed
GST pull-down assays with the point-mutated $\alpha$-SYN. $\alpha$-SYN binding to HSC70 was significantly decreased by dephosphorylation of Y136, although tyrosine phosphorylation of $\alpha$-SYN was decreased not only by Y136A but also by Y125A (Fig. 2B) but phosphorylation of $\alpha$-SYN at Y125 has not effect on the binding between $\alpha$-SYN and HSC70 and therefore we did not concern about Y125. The intensity of each band was measured using Image $\mathrm{J}$, and the results are shown in Fig. 2C.

\subsection{PTP1B inhibitor regulated the phosphorylation of focal adhesion kinase (FAK) and $\alpha$-SYN}

To search for a kinase that phosphorylates $\alpha$-SYN, we used protein interaction prediction sites [PIPS (http://www.compbio.dundee.ac.uk/www-pips/) or PRISM (http://prism.ccbb.ku.edu. tr/prism/)]. The results included CAM kinase II, protein kinase C, Fyn kinase, pp58, tyrosine kinase c-Src, FAK, pp60 (src), MKNK2 and the receptor for activated $\mathrm{C}$ kinase (RACK1).

Then, we confirmed that in SH-SY5Y cells, the phosphorylation of FAK is increased by treatment with PTP1B inhibitor, a specific membrane-permeable inhibitor of tyrosine phosphatase PTP1B $(50 \mu \mathrm{M})$, in a time-dependent fashion [19]. In previous reports, it has been shown that the activity of FAK is regulated by phosphorylation of tyrosine 925 [20]. To evaluate the FAK activity, we used an anti-phospho FAK antibody directed against tyrosine 925 . Phosphorylation of tyrosine 925 on FAK was markedly increased after a 30 min treatment with PTP1B inhibitor (Fig. 3A and B). Also, the phosphorylation of high molecular weights of $\alpha$-SYN was increased
A

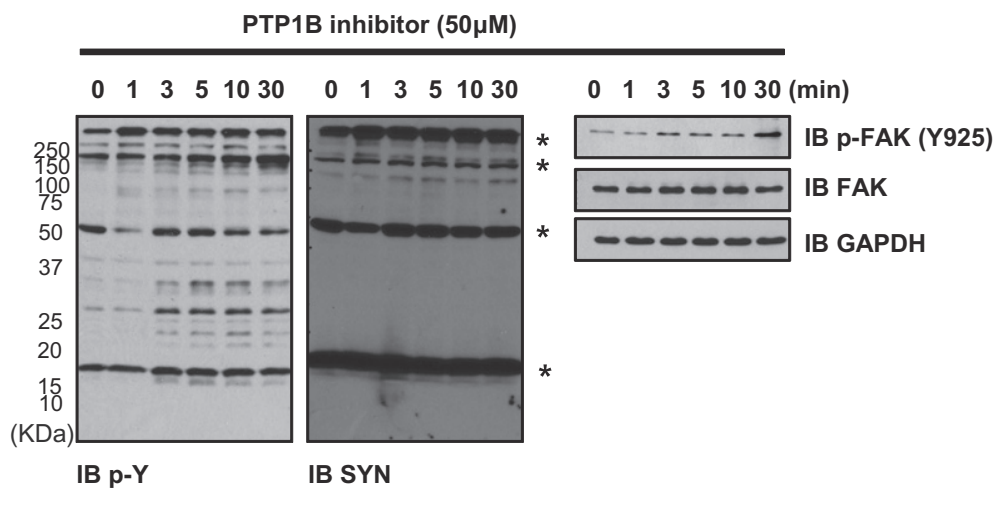

C

$\begin{array}{ll}\text { IP SYN } & \text { IP SYN } \\ \text { IB SYN } & \text { IB p-Y }\end{array}$

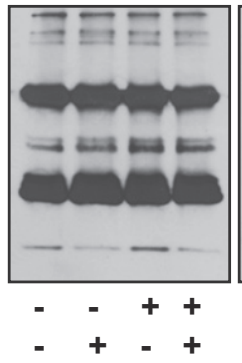

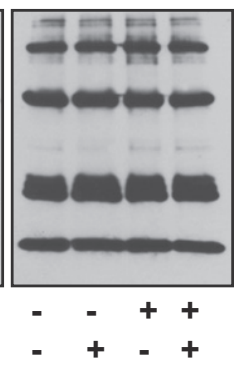

B
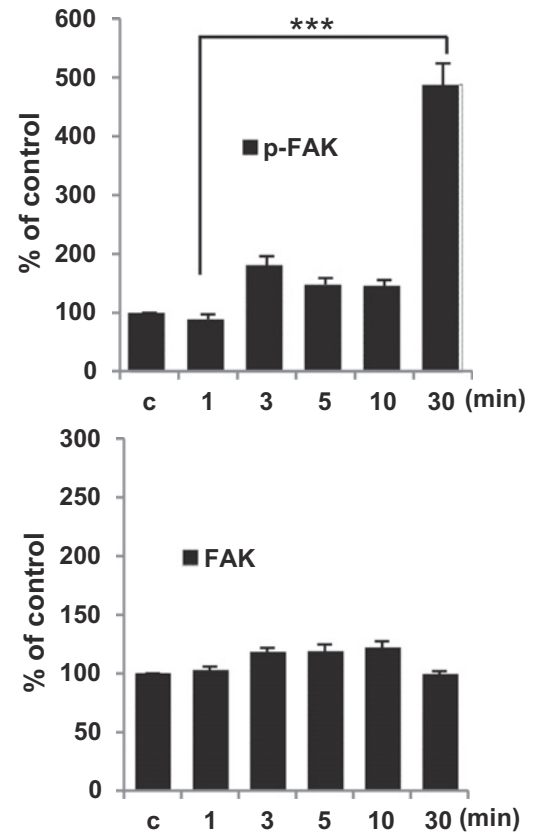





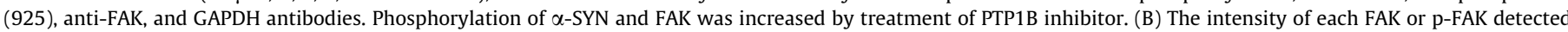



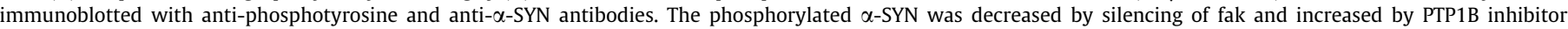
treatment. In fak silenced SH-SY5Y, the phosphorylation of $\alpha$-SYN was not induced by PTP1B inhibitor treatment. $P<0.001\left({ }^{* * *}\right)$. 
by PTP1B inhibitor in a time-dependent manner (Fig. 3A). To identify of FAK as the kinase responsible for $\alpha$-SYN phosphorylation, phosphorylated $\alpha$-SYN was immunoprecipitated in the fak silenced or PTP1B inhibitor ( $50 \mu \mathrm{M}, 30 \mathrm{~min})$ treated SH-SY5Y cell lysates and immunoblotted with anti-phosphotyrosine and anti- $\alpha$-SYN antibodies. The phosphorylated $\alpha$-SYN was decreased by knock down of fak while the phosphorylated $\alpha$-SYN was increased by PTP1B inhibitor. And, in fak silenced SH-SY5Y, PTP1B inhibitor could not induce the phosphorylation of $\alpha$-SYN (Fig. 3C).

\subsection{The effects of the PTP1B inhibitor in PQ-induced PD mouse model and SH-SY5Y cells}

To determine the effects of the PTP1B inhibitor in a PD mouse model, PQ and PTP1B inhibitor were injected into the C57BL6 mouse. The mice were divided into three groups: the control group (CTL), the PQ-only injected group (PQ) and the PQ- and PTP1B inhibitor-injected group (PQ/PTP1B inhibitor). PQ and PTP1B inhibitor were administered by i.p. injection for 3 weeks, and the behavioral test was performed in the 4 th week. First, using immunohistochemistry, we determined if the PTP1B inhibitor had a protective effect in the dopaminergic neurons. The immunohistochemical analysis showed that TH-positive cells were significantly decreased in the PQ-injected group compared to the control group and were rescued in the PTP1B inhibitor-injected group (Fig. 4A). Fig. 4B showed the measurement by Image J. In addition, we confirmed the effect of the PTP1B inhibitor in behavioral performance using the rotarod test and the elevated body swing test. Rotarod performance was significantly improved in the PTP1B inhibitor-injected group (Fig. 4C) but performance on the elevated body swing test was not. Moreover, to identify the level of $\alpha$-SYN and TH in midbrain tissue lysate, we performed Western blot analysis. The increased level of $\alpha$-SYN was decreased and the decreased level of TH was recovered by PTP1B inhibitor (Fig. 4D). Furthermore, we performed cell viability assays to confirm whether the PTP1B inhibitor has a protective effect against cytotoxicity. SH-SY5Y cells were treated with PQ $(1 \mathrm{mM})$ in the presence of the PTP1B inhibitor $(0,1,5,10 \mu \mathrm{M})$ and incubated for $24 \mathrm{~h}$. The viability of the cells was dose-dependently increased by co-treatment with the PTP1B inhibitor (Fig. 4E).

\section{Discussion}

Taken together, our experiments suggest a new direction for PD therapeutic targets through the regulation of $\alpha$-SYN phosphorylation
A

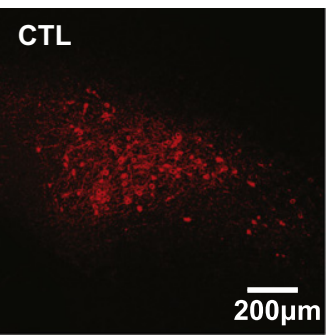

$\mathbf{P Q}$

C

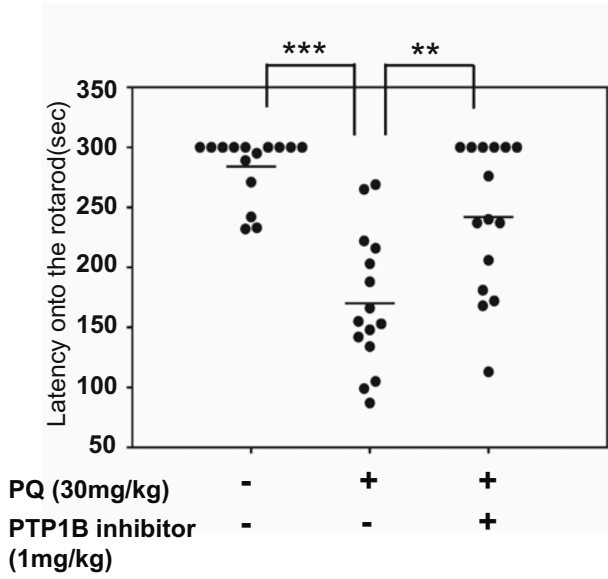

PQ / PTP1B inhibitor

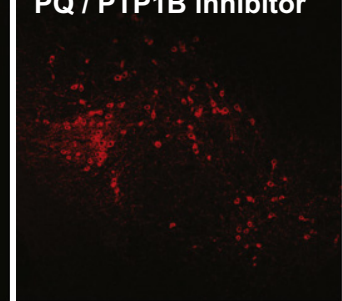

B

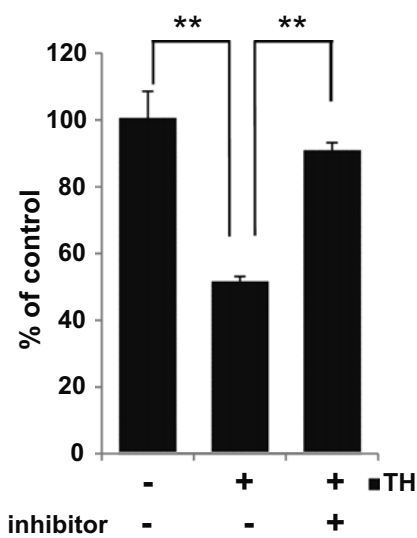

E
D

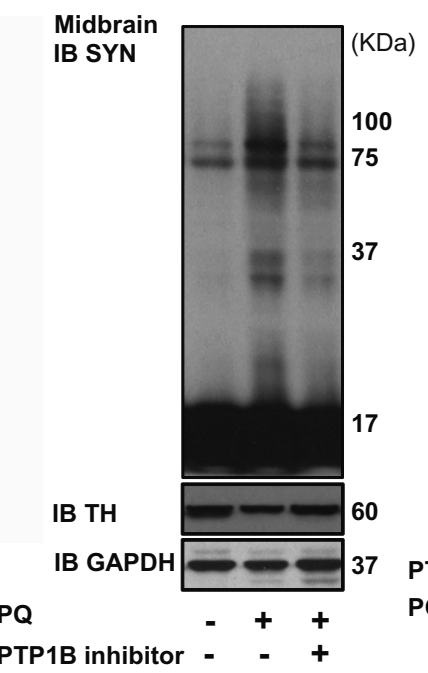

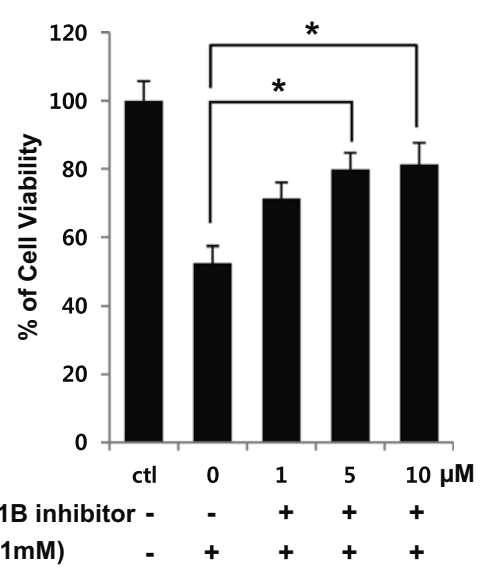

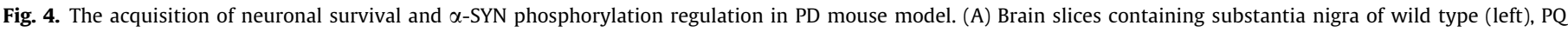

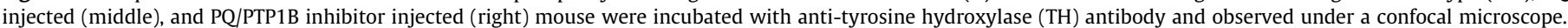

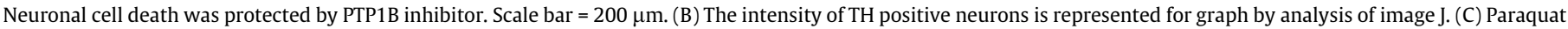

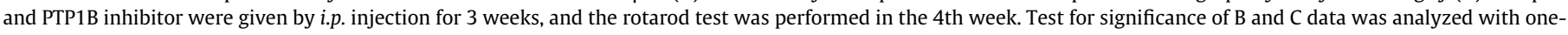

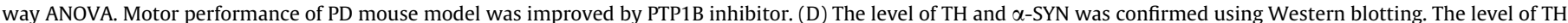

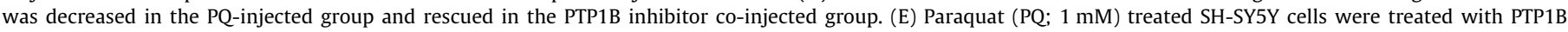

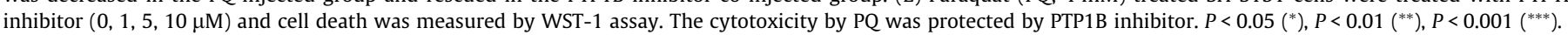


at Y136. Briefly, ubiquitinated $\alpha$-SYN is normally degraded by the proteasome, but during proteasomal dysfunction, $\alpha$-SYN can be degraded by the lysosome by binding Y136 phosphorylated $\alpha$-SYN to HSC70.

Many studies have already reported that $\alpha$-SYN can be degraded by the UPS and the CLS [21,22]. We found that when the UPS was blocked by treatment with MG132, lysosomes were activated, $\alpha$-SYN could translocate into activated lysosomes and the binding of $\alpha$-SYN to HSC70 was increased. In addition, activation of the lysosomal degradation pathway by starvation resulted in an increased binding of $\alpha$-SYN to HSC70, which was rapidly degraded by the CLS.

The key modifications that determine the binding between $\alpha$-SYN and HSC70 have not been well characterized. The pentapeptide binding motif in the $\alpha$-SYN sequence has been studied $[4,23]$. To elucidate the crucial factors involved in the degradation pathway of $\alpha$-SYN, we determined if phosphorylation of tyrosine residues on $\alpha$-SYN regulates its binding to HSC70. In our study, the binding of $\alpha$-SYN to HSC70 was increased when $\alpha$-SYN was tyrosine phosphorylated. These results suggest that tyrosine phosphorylation of $\alpha$-SYN is essential for regulating the binding of $\alpha$-SYN to HSC70. Many studies have shown that the 4 tyrosine residues (Tyr39, Tyr125, Tyr133 and Tyr136) of $\alpha$-SYN can be phosphorylated $[15,16]$. Our point mutation study also indicated that among the four tyrosine residues, Y136 in the C-termini of $\alpha$-SYN is most important for $\alpha$-SYN binding to HSC70. In human and mouse brains, $\alpha$-SYN aggregates contain the C-terminally truncated form [24]. Additionally, multiple tyrosine phosphorylations in the C-terminal segment of $\alpha$-SYN prevent eosin-induced oligomerization [7]. Therefore, it has been proposed that the C-terminus of $\alpha$-SYN may have an important role in its clearance mechanisms.

Recently, the importance of tyrosine phosphorylation of $\alpha$-SYN in the regulation of many cellular processes has been highlighted. Phosphorylation at tyrosine residues affects neurotoxicity and oligomer formation $[7,18,25]$. Some research has reported that the tyrosine residues of $\alpha$-SYN are phosphorylated by members of the Src family of protein-tyrosine kinases (PTKs) such as Fyn, cSrc and Syk $[7,15]$. Prior to kinase/phosphatase study, we predicted that FAK can be associated with $\alpha$-SYN using protein interaction prediction sites. Even in other predicted kinases, most of them are in the Src kinase family, and in this study, we examined Srcassociated phosphatases using inhibitors of SHP-1/SHP-2, PTP1B, CD45, PTP $\alpha$, LAR, CDC 25 and PTPN1. Among them, PTP1B inhibitor was effective for phosphorylation of FAK and $\alpha$-SYN and we selected PTP1B inhibitor as the target phosphatase inhibitor.

In this study, the level of phosphorylated $\alpha$-SYN was decreased in the fak silenced SH-SY5Y cells and PTP1B inhibitor cannot induce the phosphorylation of $\alpha$-SYN without FAK. According to the results, we suggest that FAK is directly related to the phosphorylation of $\alpha$-SYN and retainment of FAK activity by PTP1B inhibitor brought about the phosphorylation of $\alpha$-SYN, increased binding of $\alpha$-SYN to HSC70 and continuous movement into lysosomal compartment of $\alpha$-SYN. And the long-term treatment with PTP1B inhibitor increased neuronal survival in PQ-treated SH-SY5Y cells or in a PQ-induced PD mouse model and also conspicuously decreased the level of high molecular weight $\alpha$-SYN in the midbrain tissue. Moreover, motor balance and coordination of the mice was improved in the PTP1B inhibitor-injected group.

In summary, tyrosine phosphorylation of $\alpha$-SYN increased the binding of $\alpha$-SYN to HSC70. Among several phosphorylation sites, tyrosine Y136 in $\alpha$-SYN was the most influential for binding between two molecules. Tyrosine phosphorylation of $\alpha$-SYN was increased by PTP1B inhibitor. Increased binding of Y136 of $\alpha$-SYN to HSC70 enhanced neuronal survival in PQ treated SHSY5Y cells and PQ induced PD mouse model through PTP1B inhibitor.
Therefore, the PTP1B inhibitor might potentially be used as a therapeutic agent against PD.

\section{Acknowledgments}

This work was supported by the Conversing Research Center Program through the National Research Foundation of Korea (NRF) funded by the Ministry of Education, Science and Technology (2009-0082268), the Grant (2009K001252) from Brain Research Center of the 21st Century Frontier Research Program funded by the Ministry of Education, Science and Technology, and Seoul R\&BD Program (ST090843M0209721) by the Korean government.

\section{References}

[1] M. Goedert, Familial Parkinson's disease. The awakening of alpha-synuclein Nature 388 (1997) 232-233.

[2] M.C. Bennett, J.F. Bishop, Y. Leng, P.B. Chock, T.N. Chase, M.M. Mouradian, Degradation of alpha-synuclein by proteasome, J. Biol. Chem. 274 (1999) 33855-33858.

[3] J.L. Webb, B. Ravikumar, J. Atkins, J.N. Skepper, D.C. Rubinsztein, Alphasynuclein is degraded by both autophagy and the proteasome, J. Biol. Chem. 278 (2003) 25009-25013.

[4] A.M. Cuervo, L. Stefanis, R. Fredenburg, P.T. Lansbury, D. Sulzer, Impaired degradation of mutant alpha-synuclein by chaperone-mediated autophagy, Science 305 (2004) 1292-1295.

[5] T. Pan, S. Kondo, W. Le, J. Jankovic, The role of autophagy-lysosome pathway in neurodegeneration associated with Parkinson's disease, Brain 131 (2008) 1969-1978.

[6] K. Tanaka, T. Suzuki, N. Hattori, Y. Mizuno, Ubiquitin, proteasome and parkin, Biochim. Biophys. Acta 1695 (2004) 235-247.

[7] A. Negro, A.M. Brunati, A. Donella-Deana, M.L. Massimino, L.A. Pinna, Multiple phosphorylation of alpha-synuclein by protein tyrosine kinase Syk prevents eosin-induced aggregation, FASEB J. 16 (2002) 210-212.

[8] L. Chen, M.B. Feany, Alpha-synuclein phosphorylation controls neurotoxicity and inclusion formation in a Drosophila model of Parkinson disease, Nat. Neurosci. 8 (2005) 657-663.

[9] S.R. Paik, H.J. Shin, J.H. Lee, Metal-catalyzed oxidation of alpha-synuclein in the presence of copper(II) and hydrogen peroxide, Arch. Biochem. Biophys. 378 (2000) 269-277

[10] B.I. Giasson, J.E. Duda, I.V. Murray, Q. Chen, J.M. Souza, H.I. Hurtig, H Ischiropoulos, J.Q. Trojanowski, V.M. Lee, Oxidative damage linked to neurodegeneration by selective alpha-synuclein nitration in synucleinopathy lesions, Science 290 (2000) 985-989.

[11] Z. Yu, X. Xu, Z. Xiang, J. Zhou, Z. Zhang, C. Hu, C. He, Nitrated alpha-synuclein induces the loss of dopaminergic neurons in the Substantia nigra of rats, PLoS One 5 (2010) e9956.

[12] V. Dorval, P.E. Fraser, Small ubiquitin-like modifier (SUMO) modification of natively unfolded proteins tau and alpha-synuclein, J. Biol. Chem. 281 (2006) 9919-9924.

[13] H. Shimura, M.G. Schlossmacher, N. Hattori, M.P. Frosch, A. Trockenbacher, R. Schneider, Y. Mizuno, K.S. Kosik, D.J. Selkoe, Ubiquitination of a new form of alpha-synuclein by parkin from human brain: implications for Parkinson's disease, Science 293 (2001) 263-269.

[14] T. Nonaka, T. Iwatsubo, M. Hasegawa, Ubiquitination of alpha-synuclein, Biochemistry 44 (2005) 361-368.

[15] C.E. Ellis, P.L. Schwartzberg, T.L. Grider, D.W. Fink, R.L. Nussbaum, Alphasynuclein is phosphorylated by members of the Src family of protein-tyrosine kinases, J. Biol. Chem. 276 (2001) 3879-3884.

[16] T. Nakamura, H. Yamashita, T. Takahashi, S. Nakamura, Activated Fyn phosphorylates alpha-synuclein at tyrosine residue 125 , Biochem. Biophys. Res. Commun. 280 (2001) 1085-1092.

[17] M.K. Mbefo, K.E. Paleologou, A. Boucharaba, A. Oueslati, H. Schell, M. Fournier D. Olschewski, G. Yin, M. Zweckstetter, E. Masliah, P.J. Kahle, H. Hirling, H.A. Lashuel, Phosphorylation of synucleins by members of the Polo-like kinase family, J. Biol. Chem. 285 (2010) 2807-2822.

[18] L. Chen, M. Periquet, X. Wang, A. Negro, P.J. McLean, B.T. Hyman, M.B. Feany, Tyrosine and serine phosphorylation of alpha-synuclein have opposing effects on neurotoxicity and soluble oligomer formation, J. Clin. Invest. 119 (2009) 3257-3265.

[19] G. Huyer, S. Liu, J. Kelly, J. Moffat, P. Payette, B. Kennedy, G. Tsaprailis, M.J Gresser, C. Ramachandran, Mechanism of inhibition of protein-tyrosine phosphatases by vanadate and pervanadate, J. Biol. Chem. 272 (1997) 843851.

[20] S. Basuroy, M. Dunagan, P. Sheth, A. Seth, R.K. Rao, Hydrogen peroxide activates focal adhesion kinase and c-Src by a phosphatidylinositol 3 kinasedependent mechanism and promotes cell migration in Caco-2 cell monolayers, Am. J. Physiol. Gastrointest. Liver Physiol. 299 (2010) G186-G195.

[21] H.J. Lee, F. Khoshaghideh, S. Patel, S.J. Lee, Clearance of alpha-synuclein oligomeric intermediates via the lysosomal degradation pathway, J. Neurosci. 24 (2004) 1888-1896. 
[22] U. Bandyopadhyay, A.M. Cuervo, Chaperone-mediated autophagy in aging and neurodegeneration: lessons from alpha-synuclein, Exp. Gerontol. 42 (2007) $120-128$.

[23] J.F. Dice, Peptide sequences that target cytosolic proteins for lysosomal proteolysis, Trends Biochem. Sci. 15 (1990) 305-309.

[24] W. Li, N. West, E. Colla, O. Pletnikova, J.C. Troncoso, L. Marsh, T.M. Dawson, P. Jakala, T. Hartmann, D.L. Price, M.K. Lee, Aggregation promoting C-terminal truncation of alpha-synuclein is a normal cellular process and is enhanced by the familial Parkinson's disease-linked mutations, Proc. Natl. Acad. Sci. USA 102 (2005) 2162-2167.

[25] J.M. Savitt, V.L. Dawson, T.M. Dawson, Diagnosis and treatment of Parkinson disease: molecules to medicine, J. Clin. Invest. 116 (2006) 1744-1754. 